\title{
Biodistribution of gold nanoparticles in BBN- induced muscle-invasive bladder cancer in mice
}

This article was published in the following Dove Press journal:

International Journal of Nanomedicine

27 October 2017

Number of times this article has been viewed

\author{
Henry M Smilowitz' \\ Lauren J Tarmu ${ }^{1-3}$ \\ Mary Melinda Sanders ${ }^{4}$ \\ John A Taylor III $^{5}$ \\ Dharamainder Choudhary ${ }^{6}$ \\ Crystal Xue ${ }^{7}$ \\ Nathaniel A Dyment ${ }^{8}$ \\ Dan Sasso' \\ Xiaomeng Deng' \\ James F Hainfeld ${ }^{10}$ \\ 'Department of Cell Biology, \\ University of Connecticut Health \\ Center, Farmington, CT, ${ }^{2}$ Department \\ of Human Behavior, College of \\ Southern Nevada, North Las Vegas, \\ ${ }^{3}$ Department of Anthropology, \\ University of Nevada, Las Vegas, NV, \\ ${ }^{4}$ Department of Anatomic Pathology, \\ University of Connecticut Health \\ Center, Farmington, CT, ${ }^{5}$ Department \\ of Urology, University of Kansas \\ Medical Center, Kansas City, KS, \\ ${ }^{6}$ Department of Surgery, University \\ of Connecticut Health Center, \\ Farmington, CT, ${ }^{7}$ George Washington \\ University School of Medicine, \\ Washington, DC, ${ }^{8}$ Department of \\ Orthopedic Surgery, University of \\ Pennsylvania, Philadelphia, PA, ${ }^{9}$ David \\ Geffen School of Medicine at UCLA, \\ Los Angeles, CA, ${ }^{10}$ Nanoprobes, Inc, \\ Yaphank, NY, USA
}

Correspondence: Henry M Smilowitz Department of Cell Biology, University of Connecticut Health Center, 263 Farmington Avenue, Farmington, CT 06030, USA

Tel + I 8606792710

Fax +l 8606793693

Emailsmilowitz@uchc.edu

\begin{abstract}
Bladder-sparing options are being developed for muscle-invasive bladder cancer in place of radical cystectomy, including the combination of chemotherapy and radiation therapy. We reasoned that improving the radiotherapy component of chemoradiation could improve the control of locally advanced disease. Previously, we showed that gold nanoparticles (AuNPs) are potent enhancers of radiation therapy. We hypothesized that if AuNPs were to preferentially localize to bladder tumors, they may be used to enhance the radiation component of muscleinvasive bladder tumor therapy. Mice were treated with the carcinogen N-butyl-N-(4-hydroxybutyl)nitrosamine (BBN) for 17, 20, and 22 weeks - long enough to induce muscle-invasive tumors. Mice were then anesthetized and injected intravenously with $1.9 \mathrm{~nm}$ AuNPs of which most were rapidly cleared from the blood and excreted after a 30-50 minute residence time in the bladder. We found AuNPs distributed throughout the bladder wall, but most of the AuNPs were associated with the stroma surrounding the tumor cells or extracellular keratin produced by the tumor cells. There were relatively few AuNPs in the tumor cells themselves. The AuNPs therefore localized to tumor-associated stroma and this tumor specificity might be useful for specific X-ray dose enhancement therapy of muscle-invasive bladder carcinomas.
\end{abstract}

Keywords: N-butyl-N-(4-hydroxybutyl)nitrosamine, BBN, muscle-invasive bladder cancer, gold nanoparticles, mouse model

\section{Introduction}

Bladder cancer is the fourth and eleventh most commonly diagnosed cancer in men and women, respectively, with over 400,000 new cases and 150,000 deaths per year worldwide. ${ }^{1,2}$ Over the last three decades, the mortality rate associated with bladder cancer has not changed considerably. At initial diagnosis, $\sim 25 \%-30 \%$ of bladder cancers are diagnosed as muscle-invasive bladder cancer (MIBC). ${ }^{1,2}$ Of the $70 \%-75 \%$ of patients initially diagnosed with noninvasive bladder cancer, many progress to MIBC despite treatments. Currently, the standard treatment for localized MIBC in most western countries is radical cystectomy. ${ }^{1,2}$

Recent interest in patients' quality of life and those patients for whom radical cystectomy is not suitable has driven an increasing interest in chemotherapy, radiotherapy, and chemoradiation. ${ }^{3-10}$ A trial published in the New England Journal of Medicine concluded that the addition of chemotherapy to radiotherapy reduced invasive cancer recurrence, as compared with radiotherapy alone, resulting in good long-term bladder function and low rates of salvage cystectomy. ${ }^{3}$ Bladder preservation with chemoradiation is no longer controversial, but further study is needed to identify which patients should be initially offered bladder-sparing chemoradiation. ${ }^{4-10}$ We hypothesize that improving the radiotherapy component of chemoradiation would further decrease cancer recurrence rates. 
The tolerance level of normal tissues is the limiting factor for radiation dose levels in bladder tumor radiotherapy. New treatment methods that increase the efficacy of radiation therapy of the bladder tumors while preserving surrounding normal tissues ${ }^{11-13}$ would be expected to improve the radiotherapy component of chemoradiation, leading to higher success rates of tumor eradication and lower rates of salvage cystectomy, thereby possibly improving patients' quality of life.

Preclinical in vivo studies have shown that gold nanoparticles (AuNPs) significantly improve the efficacy of radiation therapy of a variety of tumors. ${ }^{14-17}$ When high atomic number atoms are bombarded with relatively low energy X-rays $(<500 \mathrm{keV})$, photoelectric effect is the predominant mode of photon interaction, where incident photons eject electrons from the gold atoms, thus depositing more energy locally thereby increasing the local dose. These photoelectrons acquire a kinetic energy of the primary beam energy minus its binding energy thus determining the range the photoelectrons will have in the tissue. For $100 \mathrm{keV}$ electrons, irradiation should result in ranges of tens of microns, but on average the ejected electrons travel much shorter distances - limiting most damage to cells that have either incorporated the gold or are coated by the gold. ${ }^{18}$ Importantly, with the amounts of gold that can be delivered to tumors, the overall tumor dose can be increased significantly by a factor of 2-4 times. Administration of AuNPs prior to radiation has been shown to retard tumor growth. ${ }^{14-17}$ Increasing AuNP concentrations resulted in greater tumor ablation and longer term survival. ${ }^{14}$ Thus far, no long-term toxic side effects have been associated with AuNP administration. AuNPs $<5 \mathrm{~nm}$ have been shown to be largely filtered through the kidneys and excreted in the urine. ${ }^{14}$

This study uses a mouse model of MIBC to investigate AuNP uptake into tumor cells. It has previously been reported that internalization of AuNPs into urothelial cells depends on cell differentiation stage. ${ }^{19}$ A study by Hudoklin et al treated mice for 10 weeks with N-butyl-N-(4-hydroxybutyl) nitrosamine (BBN) ad libitum in drinking water to induce hyperplasia and dysplasia of bladder urothelium in order to investigate AuNP uptake in normal and abnormal urothelial cells. ${ }^{19}$ The study found that AuNPs accumulated preferentially in the endosomes and cytosol of partially differentiated superficial urothelial cells. ${ }^{19}$

In this study, we increased the timeframe of BBN treatment in order to induce MIBC and investigated the distribution of AuNPs throughout the bladder after an intravenous (IV) injection of $1.9 \mathrm{~nm}$ AuNPs, the same AuNPs that were used in the Hudoklin study. We show that the greatest concentrations of AuNPs are found associated with tumor stromal cells and connective tissue that surround the muscleinvasive tumors. AuNPs also appear to be associated with histologically identified extracellular keratin that is produced by BBN-induced bladder tumors in this mouse model. This distribution might have utility in tumor-specific radiation dose enhancement.

\section{Materials and methods}

All of the work and study protocols performed were approved by the University of Connecticut Health Center Animal Care and Use Committee and adhered to the rules set forth in the Guide for the Care and Use of Laboratory Animals.

\section{Initiation of bladder tumors in mice}

C57BL/6 mice were maintaind in a temperature controlled environment with 12:12 light/dark cycling and provided with unrestricted access to drinking water supplemented with 0.05\% BBN (Tokyo Chemical Industry Co Ltd, Tokyo, Japan) in order to induce bladder carcinogenesis.

\section{AuNP injections}

BBN-treated mice weighing 32-40 g were anesthetized with ketamine/xylazine $(\sim 140 \mathrm{mg} / \mathrm{kg}$ ketamine and $\sim 3 \mathrm{mg} / \mathrm{kg}$ xylazine) and injected IV via tail vein injections with $\sim 40 \mathrm{mg}$ 1.9 nm AuNPs (catalog \#1102; Nanoprobes, Inc, Yaphank, NY, USA) 17, 20, and 22 weeks after the initiation of carcinogen treatment. Previous studies showed that blood levels of the $1.9 \mathrm{~nm}$ AuNPs are $90 \%$ cleared in $\sim 10$ minutes. The time of urination following AuNP injections was observed to be $\sim 40-60$ minutes.

\section{Harvest of bladders and fixation}

One day after AuNP injections, mice were euthanized and the bladders were excised, inflated with and placed into $4 \%$ formalin in phosphate-buffered saline solution for 1 day.

\section{Histopathology}

Bladders were removed from the fixative, cut in half, positioned in cassettes, and put into $70 \%$ alcohol prior to being embedded in paraffin. Paraffin blocks were cut into $6 \mu \mathrm{m}$ thick serial sections using a microtome, stained with hematoxylin and eosin (H\&E), and gold enhanced for visualization by light microscopy.

\section{Gold enhancement}

Gold enhancement is a process of depositing gold on existing AuNPs, thus enlarging them. This enhancement was 

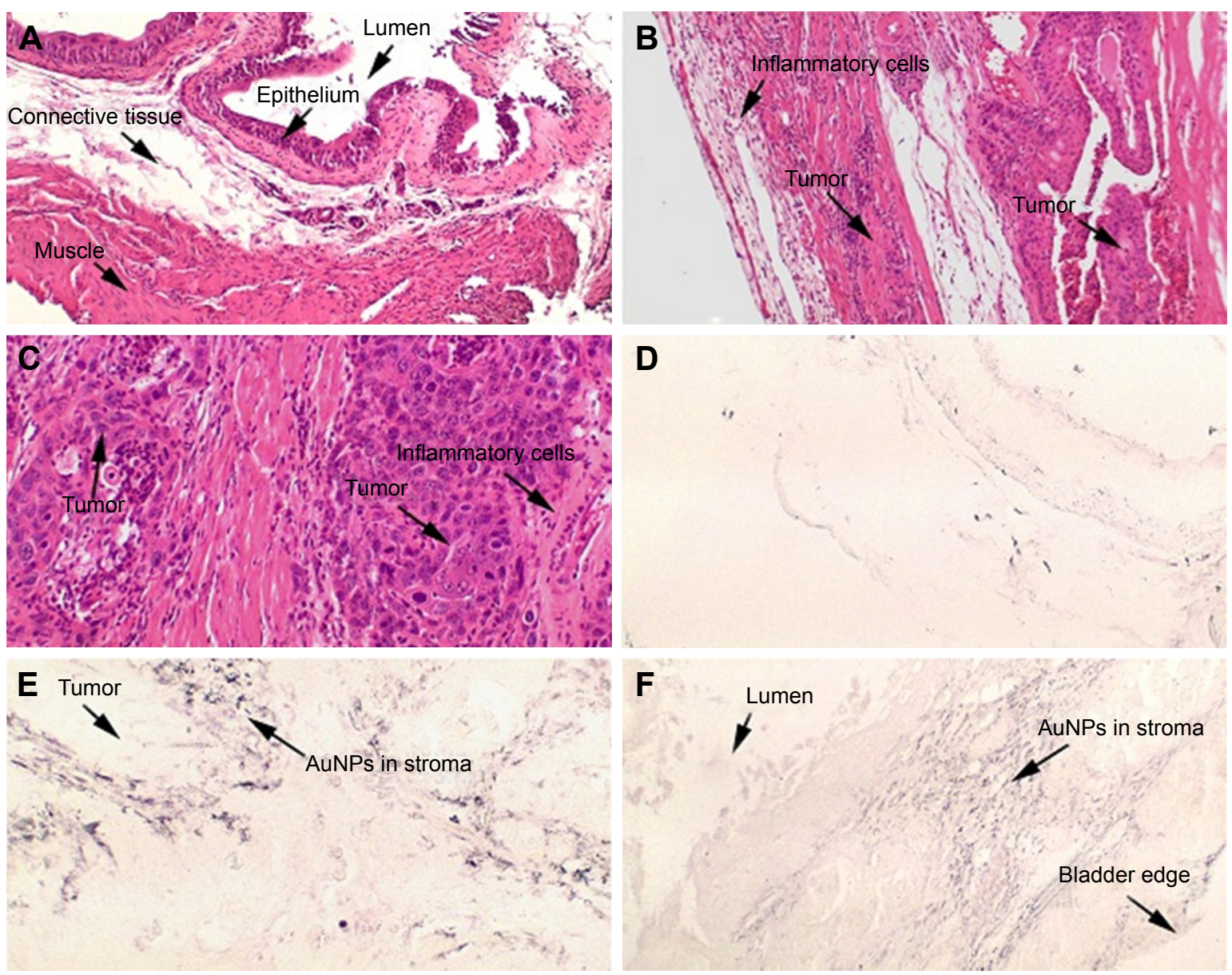

Figure I (A) Normal bladder without BBN treatment or AuNPs at 40×. (B) High-grade muscle-invasive bladder cancer induced by BBN presenting at 22 weeks at 40× and (C) at 200×. (D) Gold enhanced normal bladder without BBN treatment or AuNPs at 40× illustrates average level of background noise from gold enhancement. (E) AuNPs aggregating in the stroma surrounding tumor cells. (F) AuNPs deposit all the way out to the outer edge of the bladder enveloping tumor masses.

Abbreviations: AuNPs, gold nanoparticles; BBN, N-butyl-N-(4-hydroxybutyl)nitrosamine.

used since the tiny $1.9 \mathrm{~nm}$ AuNPs are not visible in the light microscope, but are detectable after enhancement and was performed according to the manufacturer's recommendations (Nanoprobes GoldEnhance ${ }^{\mathrm{TM}}$ LM, catalog \#2112; Nanoprobes, Inc). The time of gold enhancement was varied from 10 seconds to 10 minutes (10 seconds, 30 seconds, 60 seconds, 2 minutes, 5 minutes, 10 minutes), in order to determine the minimum exposure time that produced adequate, clear staining with low background staining. Sixty seconds allowed for most efficient visualization of AuNPs while minimizing background staining and was used for the reported studies. Subsequent to the performance of this study, it was found that backgrounds can be reduced further by transferring the slides into $15 \%(\mathrm{w} / \mathrm{v})$ sodium sulfite after a brief 1-2 minute distilled water wash.

\section{Results}

Figure $1 \mathrm{~A}$ is an H\&E stain of a section of the normal bladder from a three-month-old mouse. The lumen, epithelial, and connective tissue layers have been labeled. The thickness of the bladder wall is about $2 \mathrm{~mm}$. Ten out of eleven mice treated with BBN for 17, 20, or 22 weeks developed tumors ranging from in situ, noninvasive bladder cancer to high-grade MIBC with extensive squamous differentiation (Table 1). Six out of eleven mice developed muscle-invasive tumors (Table 2). In most cases, high inflammatory responses were seen (Figure 1B and C). AuNPs (1.9 nm AuNPs) were injected into control, untreated, and BBN-treated mice by IV injection. Previous results showed that most of these AuNPs were rapidly cleared from the blood and accumulated in the mouse bladder prior to excretion. IV injections were performed on ketamine/xylazine-anesthetized mice to delay excretion to maximize the time the AuNPs resided in the lumen of the bladder prior to excretion. AuNPs dwelled in the bladders for about 30 to 50 minutes before they were excreted. Twenty-four hours later, when the AuNPs had time to diffuse, bind, and to be taken up by cells, the mice were euthanized, the bladders were excised, inflated by injection 
Table I Histopathology of all animals in the study detailing tumor incidence

\begin{tabular}{|c|c|}
\hline Mouse & Week \\
\hline & Week I7 \\
\hline I & Cancer in submucosa and muscle; deeply muscle invasive \\
\hline 2 & $\begin{array}{l}\text { Large tumor, cancer in submucosa, muscle thinned out but } \\
\text { tumor non-muscle invasive }\end{array}$ \\
\hline 3 & No tumor, in situ disease; confined to epithelium \\
\hline 4 & Large muscle-invasive tumor \\
\hline 5 & $\begin{array}{l}\text { Lower graded lesion, cancer with no squamous } \\
\text { differentiation, tumor does not reach muscle } \\
\text { Week } 20\end{array}$ \\
\hline I & $\begin{array}{l}\text { High-grade tumor, does not reach muscle; muscle } \\
\text { attenuated with submucosa expanded by tumor }\end{array}$ \\
\hline 2 & $\begin{array}{l}\text { Extensive squamous differentiation, tumor reaches outer } \\
\text { surface of bladder extending all the way through the muscle }\end{array}$ \\
\hline 3 & $\begin{array}{l}\text { Highly inflamed, epithelium has large variable nuclei, } \\
\text { dysplastic; however, no musle-invasive tumor } \\
\text { Week } 22\end{array}$ \\
\hline 1 & $\begin{array}{l}\text { Extensive keratin and inflammation, tumor through bladder } \\
\text { wall, profound inflammatory response }\end{array}$ \\
\hline 2 & $\begin{array}{l}\text { Tumor growing down and invading the muscle, tumor stays } \\
\text { superficial in the muscle }\end{array}$ \\
\hline 3 & No muscle-invasive tumor present \\
\hline
\end{tabular}

of formalin directly into the bladder lumen, and dropped into $4 \%$ formalin for 24 hours. Fixed bladders were embedded in paraffin, sectioned, and either stained with H\&E or gold enhanced following the protocol prescribed by Nanoprobes, Inc. A pilot study revealed that 1 minute of gold enhancement best highlighted distributions of gold within the tissue. Figure 1D shows a gold enhanced normal bladder without BBN or AuNP injection and illustrates that the average level of background noise from 1 minute of gold enhancement is very low. Tissue sections from tumor containing BBN-treated bladders showed much higher levels of gold enhancement. Interestingly, tumor cells themselves generally had very little AuNP uptake (Figure 1E). In most cases, AuNPs accumulated in the stroma surrounding the tumor cells (Figures 1E, F and $2 \mathrm{~A}-\mathrm{E}$ [ 20 weeks of BBN treatment]). AuNP uptake in the stroma extended all the way out to the edge of the bladder (Figure 1F). Even in deeply invasive muscle tumors, AuNPs enveloped the tumor cell stroma but not the tumor cells themselves (Figure 1E). AuNPs were enriched in the submucosal, connective tissue space, with little AuNP uptake

Table 2 Number of mice that developed muscle-invasive tumors

\begin{tabular}{llll}
\hline & \multicolumn{3}{l}{ Group } \\
\cline { 2 - 4 } & A & B & C \\
\hline Number of mice treated with BBN & 5 & 3 & 2 \\
Number of weeks treated & 17 & 20 & 22 \\
Fraction of mice that developed muscle-invasive tumor & $3 / 5$ & $1 / 3$ & $2 / 2$ \\
\hline
\end{tabular}

Abbreviation: BBN, N-butyl-N-(4-hydroxybutyl)nitrosamine. in the epithelial layer. Regions of bladder tissue devoid of tumor cells showed few, if any, AuNPs (Figures 1D and 2F). Rarely, tumor cells with AuNPs within them were observed (Figures 2B, D, and E). Representative controls, for example, BBN treatment without AuNP injection (Figure 3A-F) and no BBN treatment with AuNP injection (Figure 4A-F), did not show remarkable gold labeling after enhancement. Such controls were performed for 17, 20, and 22-week treatment groups. Gold labeling of the bladder surface was evident. Upon reviewing all the sections under $40 \times$, we took four sample urothelium stretches and defined the amount of AuNPs in them as $0,1+, 2+$, and $3+$, respectively. Based on these references, we determined the amount of AuNPs in every bladder section and found that non-BBN treated mouse bladders had significantly more gold labeling on the surface ( $p$-value $<0.001$ ) compared to BBN-treated mice (Table 3 and Figure 5); further studies are needed to determine if the gold particles are associated with urothelium or the extracellular mucous layer. Bladder tumors in mice have greater squamous differentiation than most human bladder tumors. In this mouse model of MIBC, keratin, identified morphologically, filled the bladder lumen possibly trapping AuNPs within the keratin (Figure 6). Human bladder cancers typically do not present with such high levels of keratinization..$^{20,21}$ Figure 7A-D shows gold labeling of the tumor stroma in background-reduced (sodium sulfite-treated) gold enhanced sections. Some AuNPs are seen in non-BBNtreated AuNP injected bladders (Figure 7E and F), but they are far less numerous and less localized than in tumor containing bladders. Unfortunately, it was not possible to redo the entire study with background reduction due to lack of sample material.

\section{Discussion}

Our study was motivated by the study of Hudoklin et al whose evidence suggested that IV injected $1.9 \mathrm{~nm}$ AuNPs, nanoparticles produced by Nanoprobes Inc, are taken up selectively into dedifferentiated urothelium in mice treated for 10 weeks with the carcinogen BBN. ${ }^{19}$ We had shown previously that $90 \%$ of IV injected $1.9 \mathrm{~nm}$ AuNPs are cleared from the blood in about 10 minutes via the kidneys. ${ }^{17}$ On the basis of the Hudoklin study and our previous work, we hypothesized that $1.9 \mathrm{~nm}$ AuNPs injected IV into mice would quickly enter the bladder and remain in the bladder for a sufficiently long period of time to allow the particles to specifically enter dedifferentiated bladder tissue and, in particular, muscle-invasive bladder tumors, largely from the bladder lumen. We further hypothesized that such specific uptake of AuNPs into muscle-invasive 

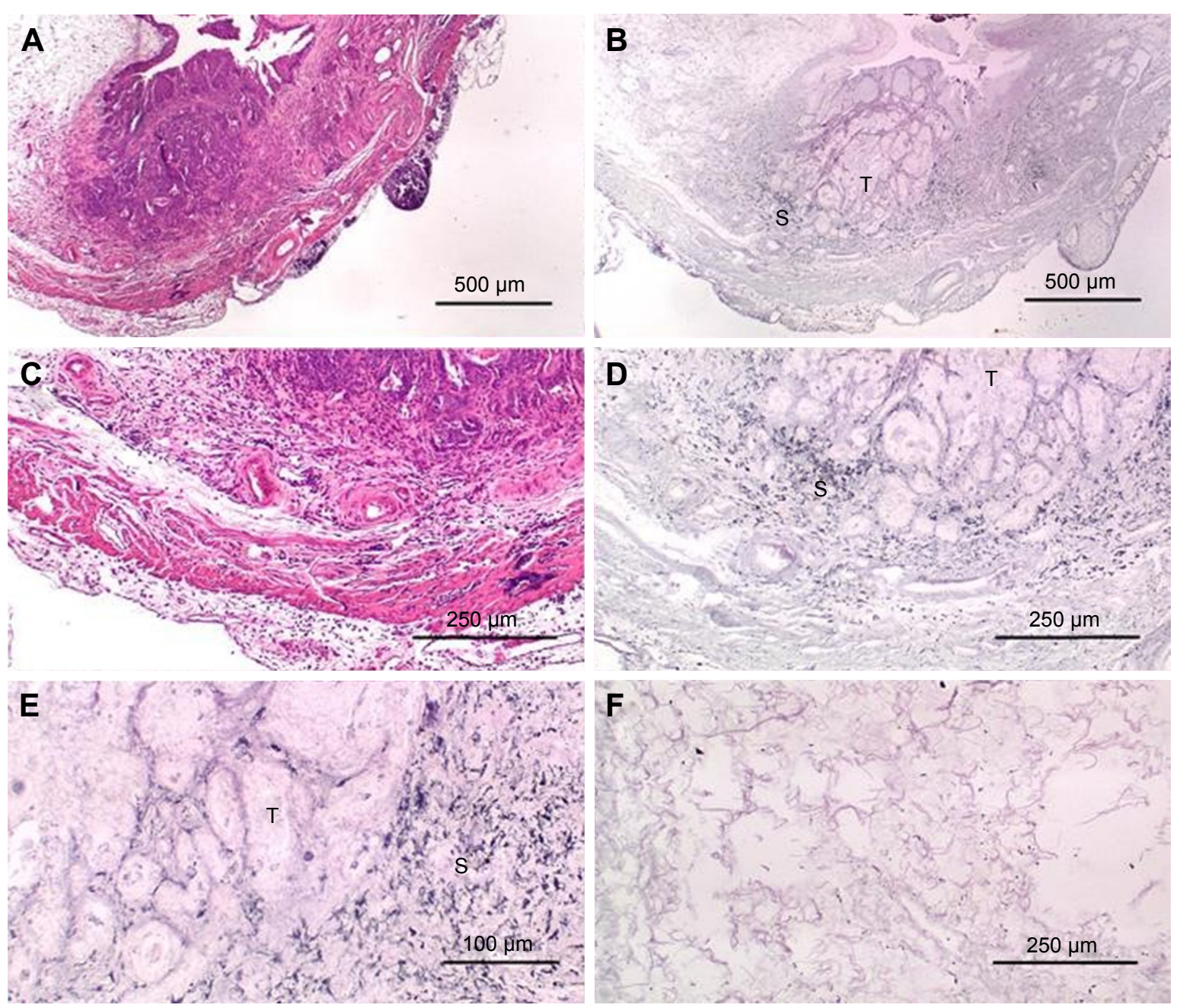

Figure 2 High-grade muscle-invasive bladder cancer induced by BBN treatment at 20 weeks. (A) H\&E, 40x; (B) gold enhanced, 40x; (C) H\&E, I00x; (D) gold enhanced, 100×; (E) gold enhanced, 200x; (F) gold enhanced region of bladder wall devoid of tumor, 100x.

Abbreviations: BBN, N-butyl-N-(4-hydroxybutyl)nitrosamine; H\&E, hematoxylin and eosin; T, tumor; S, stroma.

bladder tumors and not normal urothelium would provide therapeutic benefit as we have shown AuNPs are powerful enhancers of radiotherapy. ${ }^{14-16}$ As a first step towards testing these hypotheses, mice were treated with BBN for a longer time period than in the Hudoklin study, that is, 17, 20, and 22 weeks - sufficient time for muscle-invasive tumors to form. Indeed, muscle-invasive bladder tumors were found in six of the ten mice that developed bladder tumors, whereas after 10 weeks of BBN treatment, Hudoklin did not report any muscle-invasive tumors. AuNPs injected IV into mice indeed largely remained in the bladder for $\sim 30$ to 50 minutes prior to urination.

Histological investigation of the bladders revealed that while some AuNPs could be found throughout the bladders, most of the AuNPs could be found localized to the connective tissue and stroma surrounding the tumor cells rather than in and around the tumor cells themselves
(Figures 1-4). In many instances, cells in the stroma appear to have taken up the gold. A more detailed study of the distribution of the gold in the tumor stroma will be done in future studies to identify the cells and specific structures accumulating the AuNPs. In general, 1) regions of the bladder devoid of tumor generally had relatively few AuNPs (Figure 2F); 2) bladders from BBN-treated mice that did not receive IV gold injections showed a low level of enhancement (Figure 3) indicating that the gold enhancement treatment by itself could not explain the extensive labeling of the stroma seen in bladders of BBN-treated mice that received IV gold injections; 3) while bladders from non-BBN-treated mice that received IV gold injections had gold labeling (Figure 4), most of the gold labeled the epithelium; connective tissue labeling was less intense than that seen in BBN and gold-treated mice; 4) bladders of normal, non-tumor bearing animals did not 

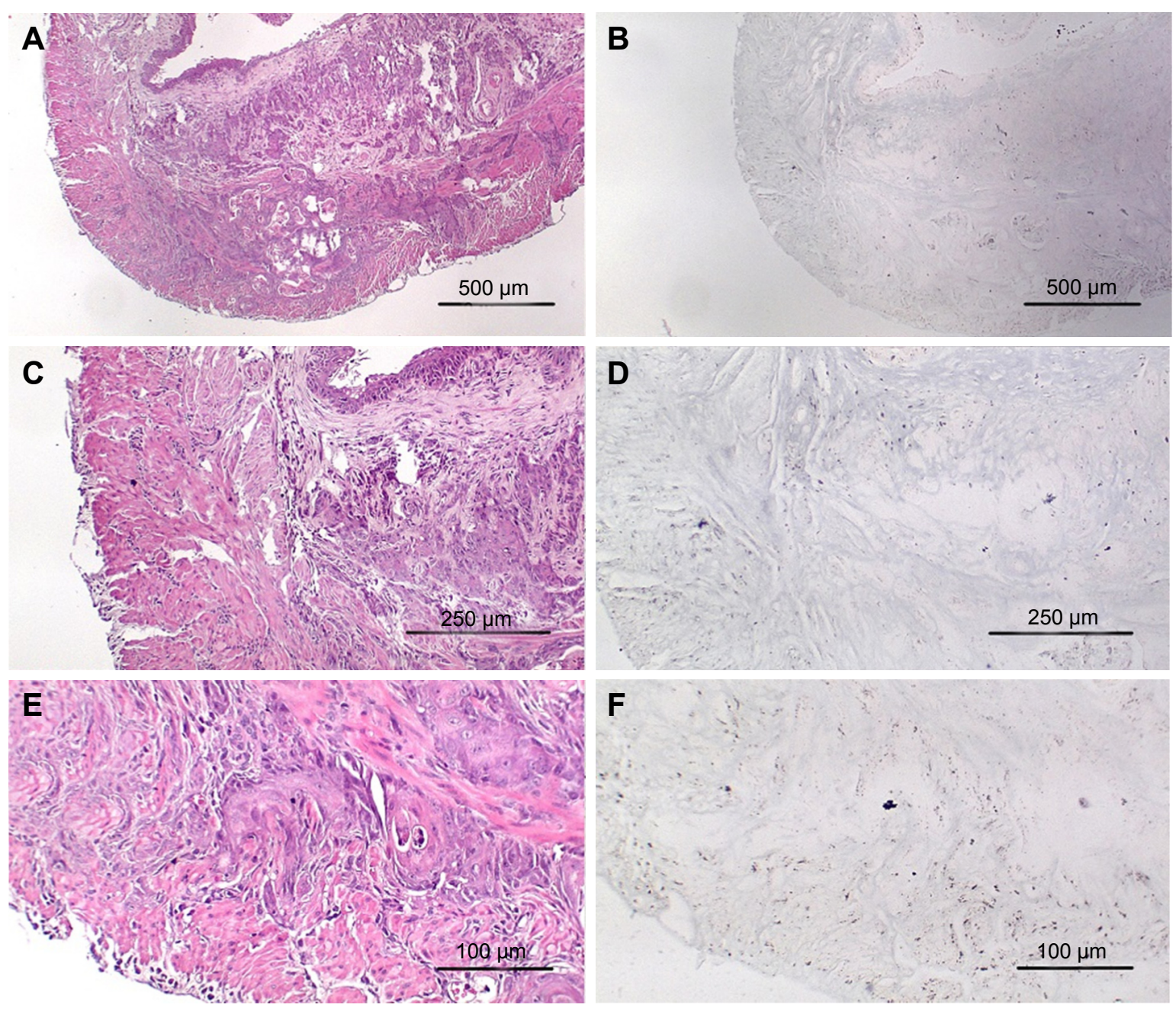

Figure 3 High-grade muscle-invasive bladder cancer induced by BBN treatment at 17 weeks without gold injection. (A) H\&E, 40×; (B) gold enhanced, 40×; (C) H\&E, I00×; (D) gold enhanced, I00×; (E) H\&E, 200×; (F) gold enhanced, 200×.

Abbreviations: H\&E, hematoxylin and eosin; BBN, N-butyl-N-(4-hydroxybutyl)nitrosamine.

have the greatly expanded connective tissue regions seen in those with MIBC; 5) the most intense gold labeling was found over morphologically identified keratin fibers. We hypothesize that keratin-filled bladder lumen either trapped AuNPs within the keratin or bound to the AuNPs (Figure 6). Human bladder cancers typically do not present with such high levels of keratinization. ${ }^{20,21}$ Questions are therefore raised as to what the gold distributions would be like in the absence of keratin in human transitional cell bladder tumors and how much of the gold found in the connective tissue of BBN-treated mouse bladders was bound to intracellular keratin.

In this study, the AuNPs were injected IV. Some of the AuNPs probably entered the bladder tumors via leaky vessels in the vicinity of the bladder tumors by the enhanced permeability and retention effect. ${ }^{22}$ Since blood vessels are found throughout the stromal region, such AuNPs would have ready access to stromal structures and cells for binding and uptake.
AuNPs that accumulate in the bladder lumen may enter the bladder by pinocytosis and endocytosis mechanisms ${ }^{23,24}$ where the urothelium is intact. However, since there are likely to be regions of necrosis and ulceration due to the tumor, it is likely that AuNP leakage into the bladder occurred where the urothelium was abrogated. Once inside the bladder, AuNPs appear to selectively bind to connective tissue and to be taken up by stromal cells. Uptake of AuNPs by macrophage and other cells found in the tumor stroma, like fibroblasts, is well documented. ${ }^{25}$

Accumulation of untargeted AuNPs into stromal cells surrounding tumor cells could provide a therapeutic opportunity since the tumor stroma is known to affect the progression of many tumors, ${ }^{26}$ including urothelial cancer. ${ }^{27}$ The accumulation of large amounts of nanoparticles into tumor stromaassociated cells would render them much more sensitive to radiation therapy. Macrophage in tumors, generally known as tumor-associated macrophage (TAMS), are often upregulated 

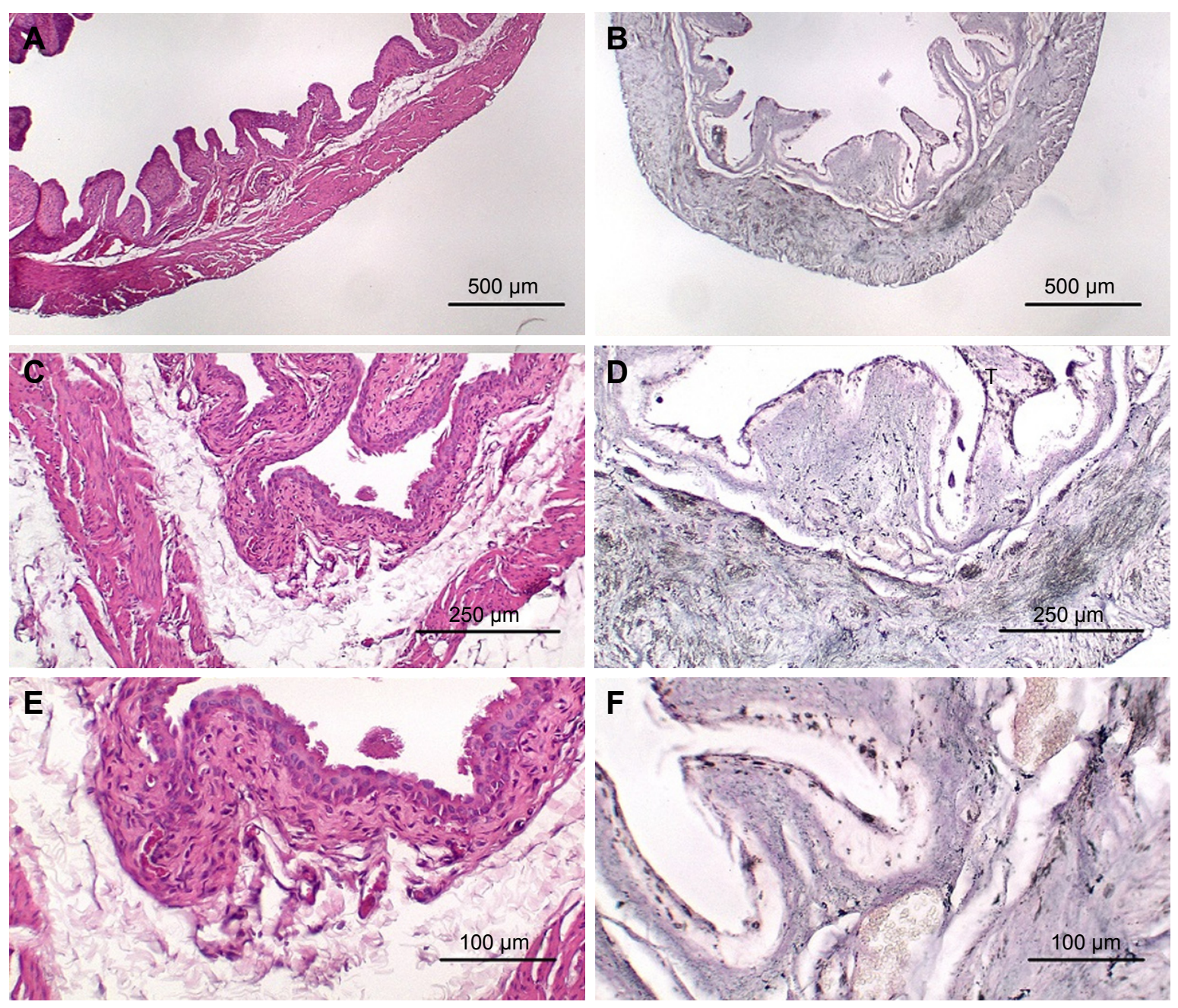

Figure 4 Bladder from age matched mouse that was not treated with BBN but received AuNP injection. (A) H\&E, 40×; (B) gold enhanced, 40×; (C) H\&E, I00×; (D) gold enhanced, 100×; (E) H\&E, 200×; (F) gold enhanced, 200×.

Abbreviations: AuNP, gold nanoparticle; H\&E, hematoxylin and eosin; BBN, N-butyl-N-(4-hydroxybutyl)nitrosamine.

in the stromal compartment of solid tumors and participate in their growth, angiogenesis, and metastasis. ${ }^{26}$ Activated cancer-associate fibroblasts can also promote tumor growth, invasion, and metastasis. ${ }^{28}$ AuNPs are known to be taken up into TAMS..$^{25}$ The selective destruction of tumor associated stromal cells could affect not only tumor proliferation, angiogenesis, and metastasis but also immune suppression ${ }^{29}$ and the efficacy of drug delivery and chemotherapy ${ }^{30}$ Hence, it is important to identify the stromal cells that take up the AuNPs and to understand their heightened sensitivity to radiation therapy. In addition, targeting of AuNPs to the tumor cells might result in greater tumor cell uptake, ${ }^{31}$ thus rendering the tumor cells themselves more sensitive to radiation therapy.

It would be of interest to determine the distribution of AuNPs after their direct introduction into the bladder compared to the IV injection of $20 \mathrm{~nm}$ AuNPs that are too large to be filtered by the kidney. ${ }^{32,33}$ In humans, direct instillation of the gold into the bladder would be possible through the urethra. Since the BBN model only produces tumors in male mice, ${ }^{34}$ direct instillation of AuNPs into the bladders of male mice is challenging, but could be done surgically. Further, transitional human bladder tumors could be injected into

Table 3 Comparison of the abundance of AuNPs in the urothelium of control and BBN-treated bladders

\begin{tabular}{lllll}
\hline Week & Group & $\begin{array}{l}\text { Mean level } \\
\text { of AuNP }\end{array}$ & $\begin{array}{l}\text { Standard } \\
\text { error }\end{array}$ & $\begin{array}{l}\boldsymbol{p} \text {-value of } \\
\text { Student's t-test } \\
\text { (vs control) }\end{array}$ \\
\hline Week 17 & Control & 2.373 & 0.066 & - \\
& BBN group I & 1.591 & 0.079 & $<0.00 I^{*}$ \\
& BBN group 2 & 0.761 & 0.068 & $<0.00 I^{*}$ \\
Week 20 & Control & 2.007 & 0.072 & - \\
& BBN group & 0.874 & 0.082 & $<0.00 I^{*}$ \\
Week 22 & Control & 1.565 & 0.109 & - \\
& BBN group & 0.375 & 0.064 & $<0.00 I^{*}$ \\
\hline
\end{tabular}

Note: *Significant.

Abbreviations: AuNP, gold nanoparticle; BBN, N-butyl-N-(4-hydroxybutyl)nitrosamine. 


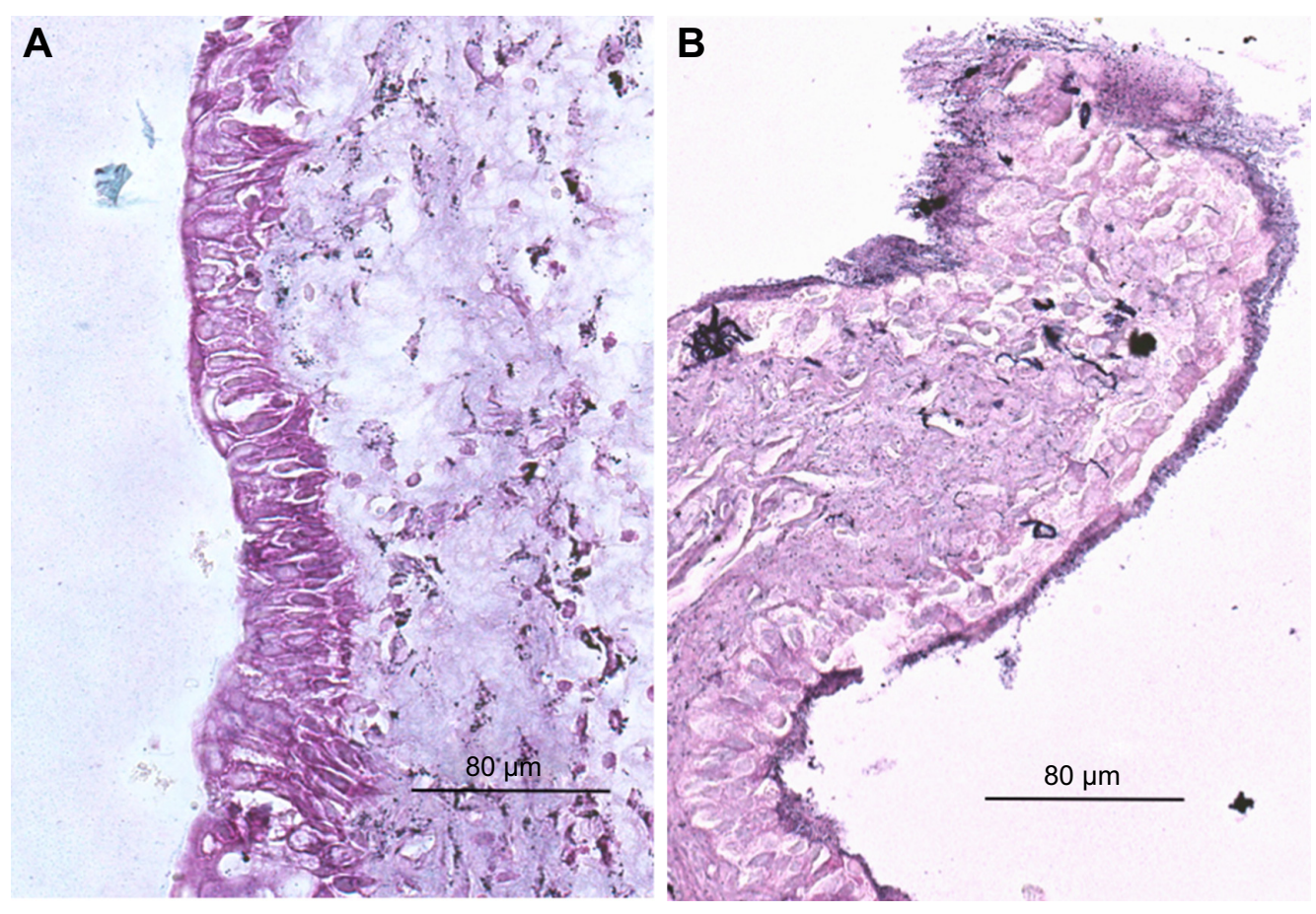

Figure 5 Representative urothelial regions in bladders of mice that received AuNP injection. (A) BBN-treated, gold enhanced, 40×; (B) non-BBN-treated, gold enhanced, 40X. Abbreviations: AuNP, gold nanoparticle; BBN, N-butyl-N-(4-hydroxybutyl)nitrosamine.
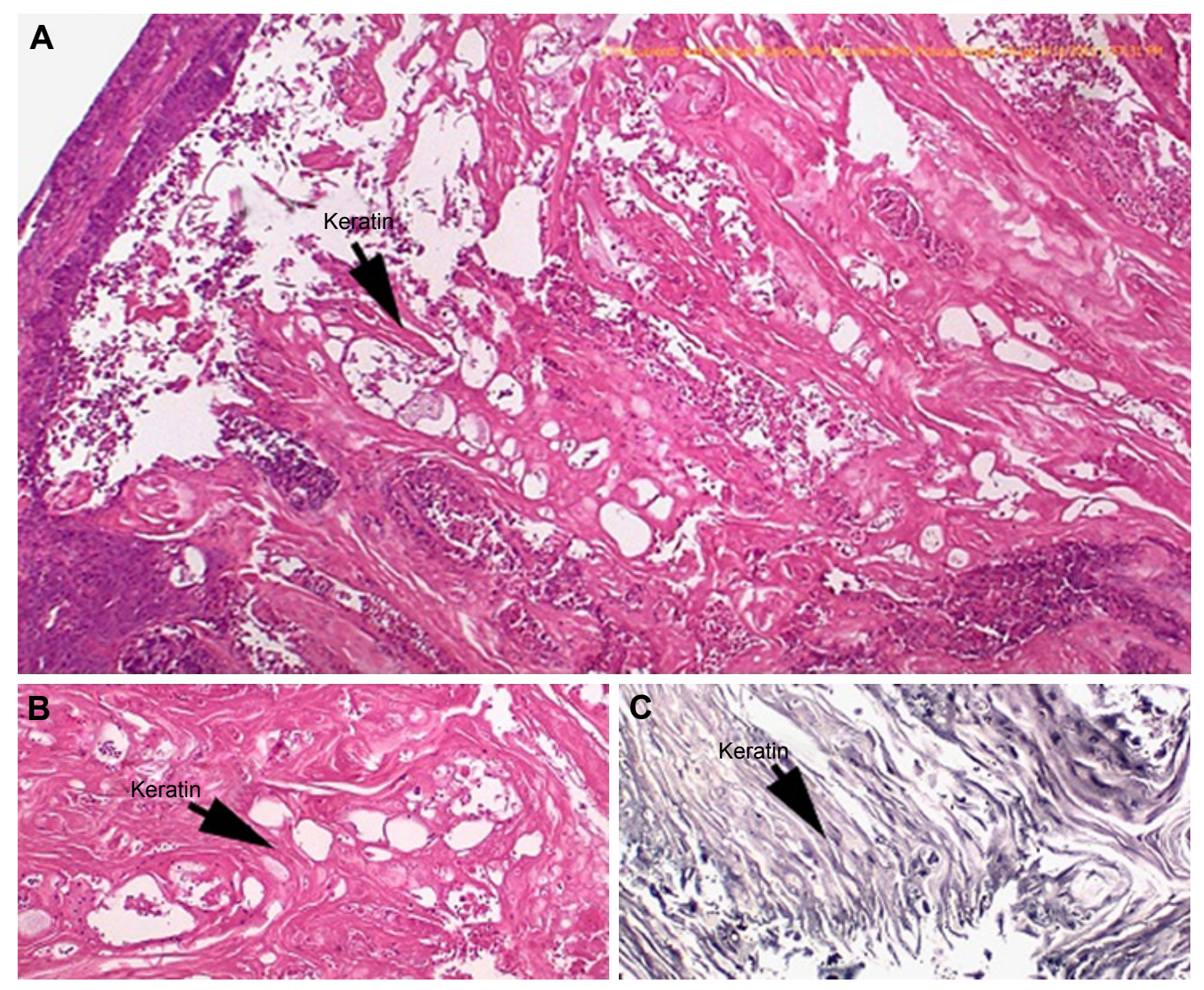

Figure 6 Keratin in the lumen of bladder from a mouse treated with BBN and stained with H\&E. (A) 40x; (B) 100x; (C) gold enhanced, 200x. Abbreviations: $\mathrm{H} \& E$, hematoxylin and eosin; BBN, N-butyl-N-(4-hydroxybutyl)nitrosamine. 

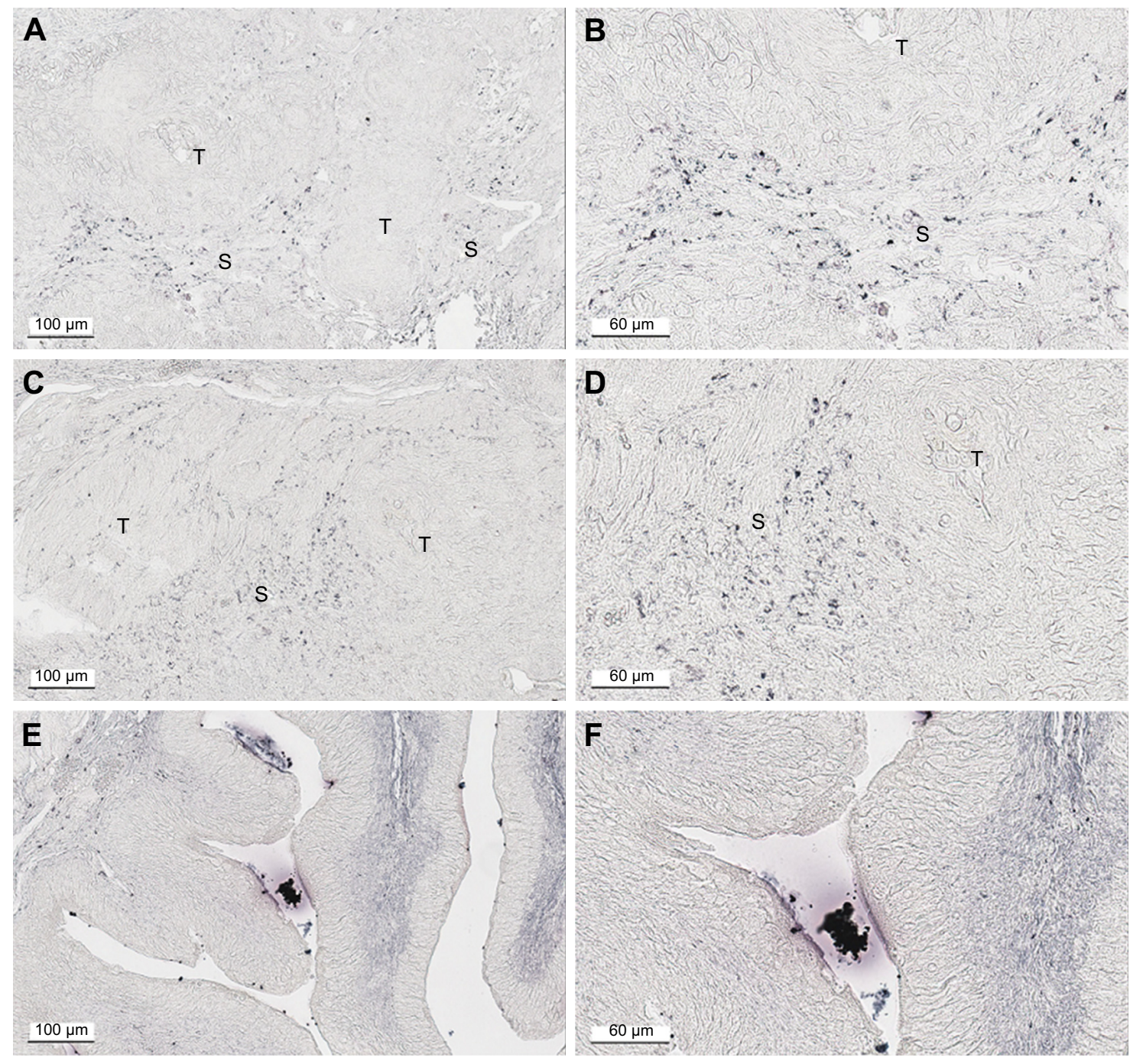

Figure 7 Low background AuNP distribution in bladders from untreated control and BBN-treated mice. (A, B) BBN, I7 weeks; (C, D) BBN, 22 weeks; (E, F) control untreated, 22 weeks.

Abbreviations: AuNP, gold nanoparticle; BBN, N-butyl-N-(4-hydroxybutyl)nitrosamine; T, tumor; S, stroma.

the wall of the athymic nude mouse bladder, estimated to be $\sim 2 \mathrm{~mm}$ thick. After tumors are thusly established, AuNP distributions could then be studied after AuNP instillation into the bladder lumen.

\section{Conclusion}

Using a model of advanced, muscle-invasive BBN-induced bladder tumors in mice, our data show that AuNPs are largely concentrated in the stroma surrounding tumor cells and significantly less in normal muscle and connective tissue of the non-tumor containing regions of the bladder. Although tumor-specific gold loading did not occur after IV AuNP administration, this mode of tumor stroma-specific loading of AuNPs might provide therapeutically useful radiation dose enhancements to enhance radiotherapy of MIBC.

\section{Acknowledgments}

We wish to thank Daniel Slatkin, MD, for his insightful ideas and inspiration over the course of this work and support from the Leo and Anne Albert Charitable Trust.

\section{Disclosure}

James Hainfeld is part owner of Nanoprobes, Inc. The other authors report no conflicts of interest in this work.

\section{References}

1. Witjes JA, Compérat E, Cowan NC, et al. Guidelines on muscle-invasive and metastatic bladder cancer. Eur Urol. 2014;65(4):778-792.

2. Kamat AM, Hahn NM, Efstathiou JA, et al. Bladder cancer. Lancet. 2016;388(10061):2796-2810

3. James ND, Hussain SA, Hall E, et al. Radiotherapy with or without chemotherapy in muscle-invasive bladder cancer. N Engl J Med. 2012; $366: 1477-1488$. 
4. Byun SJ, Kim JH, Oh YK, Kim BH. Concurrent chemoradiotherapy improves survival outcome in muscle-invasive bladder cancer. Radiat Oncol J. 2015;33(4):294-300.

5. McHaffie DR, Kruser TJ, Gaston K, Mahoney J, Graham D, Haake M. Chemoradiation for organ preservation in the treatment of muscleinvasive bladder cancer. Urol Oncol. 2016;34(6):271-278.

6. Sakaguchi M, Maebayashi T, Aizawa T, Ishibashi N, Saito T. Clinical results for bladder cancer treated by radiotherapy without concurrent standard chemotherapy. Anticancer Res. 2016;36(10):5519-5525.

7. Sargos P, Baumann BC, Eapen LJ, et al. Adjuvant radiotherapy for pathological high-risk muscle invasive bladder cancer: time to reconsider? Transl Androl Urol. 2016;5(5):702.

8. Mirza A, Choudhury A. Bladder preservation for muscle invasive bladder cancer. Bl Cancer. 2016;2(2):151-163.

9. Skinner EC. Treatment of muscle-invasive bladder cancer in older patients. Am Soc Clin Oncol. 2016;35:e228-e233.

10. Maebayashi T, Ishibashi N, Aizawa T, et al. Radiotherapy for muscleinvasive bladder cancer in very elderly patients. Anticancer Res. 2016; 36(9):4763-4769.

11. Whalley D, Caine H, McCloud P, Guo L, Kneebone A, Eade T. Promising results with image guided intensity modulated radiotherapy for muscle invasive bladder cancer. Radiat Oncol. 2015;10(1):1.

12. Takaoka EI, Miyazaki J, Ishikawa H, et al. Long-term single-institute experience with trimodal bladder-preserving therapy with proton beam therapy for muscle-invasive bladder cancer. Jpn J Clin Oncol. 2017; 47(1):67-73.

13. Lutkenhaus LJ, van Os RM, Bel A, Hulshof MC. Clinical results of conformal versus intensity-modulated radiotherapy using a focal simultaneous boost for muscle-invasive bladder cancer in elderly or medically unfit patients. Radiat Oncol. 2016;11(1):1.

14. Hainfeld JF, Slatkin DN, Smilowitz HM. The use of gold nanoparticles to enhance radiotherapy in mice. Phys Med Biol. 2004;49: N309-N315.

15. Hainfeld JF, Dilmanian FA, Slatkin DN, Smilowitz HM. Radiotherapy enhancement with gold nanoparticles. J Pharm Pharmacol. 2008; 60(8):977-985.

16. Hainfeld JF, Dilmanian FA, Zhong Z, Slatkin DN, Kalef-Ezra JA, Smilowitz HM. Gold nanoparticles enhance the radiation therapy of a murine squamous cell carcinoma. Phys Med Biol. 2010;55(11):3045.

17. Hainfeld JF, Smilowitz HM, O’Connor MJ, Dilmanian FA, Slatkin DN. Gold nanoparticle imaging and radiotherapy of brain tumors in mice. Nanomedicine. 2013;8(10):1601-1609.

18. Sung W, Ye SJ, McNamara AL, et al. Dependence of gold nanoparticle radiosensitization on cell geometry. Nanoscale. 2017;9:5843-5853.

19. Hudoklin S, Zupančič D, Makovec D, Kreft ME, Romih R. Gold nanoparticles as physiological markers of urine internalization into urothelial cells in vivo. Int J Nanomedicine. 2013;8:3945-3953.
20. Asamoto M, Fukushima S, Tatemoto Y, Yamada K, Fukui S, Mori M. Immunohistochemical expression of keratin proteins in urinary bladder carcinoma. Pathol Res Pract. 1989;184(2):194-201.

21. Herman CJ, Vegt PDJ, Debruyne FMJ, Vooijs GP, Ramaekers FCS. Squamous and transitional elements in rat bladder carcinomas induced by N-butyl-N-4-hydroxybutylnitrosamine (BBN). Am J Pathol. 1985; 120:419-426.

22. Maeda H, Nakamura H, Fang J. The EPR effect for macromolecular drug delivery to solid tumors: improvement of tumor uptake, lowering of systemic toxicity, and distinct tumor imaging in vivo. Adv Drug Deliv Rev. 2013;65:71-79.

23. Chithrani DB. Intracellular uptake, transport, and processing of gold nanostructures. Mol Membr Biol. 2010;27:299-311.

24. Baru S, Mitragotri S. Challenges associated with penetration of nanoparticle across cell and tissue barriers: a review of current status and future prospects. Nano Today. 2014;9:223-243.

25. Pal R, Chakraborty B, Nath A, et al. Noble metal-nanoparticle-induced oxidative stress modulates tumor associated macrophages (TAMS) from an M2 to M1 phenotype: an in vivo approach. Int Immunopharmacol. 2016;38:332-341.

26. Goswami K, Ghosh T, Ghosh S, Sarkar M, Bose A, Baral R. Tumor promoting role of anti-tumor macrophages in tumor microenvironment. Cell Immunol. 2017;316:1-10.

27. Hatina J, Kripnerova M, Tukova J, et al. Tumor-Stroma Interaktionen im Harnblasenkarzinom [Tumor stroma interactions in urothelial cancer]. Urologe A. 2015;54:516-525. German.

28. Ji X, Zhu X, Lu X. Effect of cancer-associated fibroblasts on radiosensitivity of cancer cells. Future Oncol. 2017;17:1537-1550.

29. Jiang W, Chan CK, Weissman IL, Kim BYS, Hahn SM. Immune priming of the tumor microenvironment by radiation. Trends Cancer. 2016;11:638-645.

30. Yang S, Gao H. Nanoparticles for modulating tumor microenvironments to improve drug delivery and tumor therapy. Pharmacol Res. Epub 2017 May 10.

31. Hainfeld JF, O'Connor MJ, Dilmanian FA, Slatkin DN, Adams DJ, Smilowitz HM. Micro-CT enables microlocalization and quantification of Her-2-targeted gold nanoparticles within tumor regions. Br J Radiol. 2011;84:526-533.

32. Zhang XD, Yang J, Song SS, et al. Passing through the renal clearance barrier: toward ultrasmall sizes with stable ligands for potential clinical applications. Int J Nanomedicine. 2014;9:2069-2072.

33. Longmire M, Choyke PL, Kobayashi H. Clearance properties of nanosized particles and molecules as imaging agents: considerations and caveats. Nanomedicine (Lond). 2008;5:703-717.

34. Overdevest JB, Knubel KH, Duex JE, et al. CD24 expression is important in male urothelial tumorigenesis and metastasis in mice and is androgen regulated. Proc Natl Acad Sci. 2012;109(51):E3588-E3596.
International Journal of Nanomedicine

\section{Publish your work in this journal}

The International Journal of Nanomedicine is an international, peerreviewed journal focusing on the application of nanotechnology in diagnostics, therapeutics, and drug delivery systems throughout the biomedical field. This journal is indexed on PubMed Central, MedLine, CAS, SciSearch ${ }^{\circledR}$, Current Contents ${ }^{\circledR} /$ Clinical Medicine,

\section{Dovepress}

Journal Citation Reports/Science Edition, EMBase, Scopus and the Elsevier Bibliographic databases. The manuscript management system is completely online and includes a very quick and fair peer-review system, which is all easy to use. Visit http://www.dovepress.com/ testimonials.php to read real quotes from published authors. 